\title{
Gender disparities in life expectancy and mortality from preventable diseases in Latvia from 2000 to 2020
}

\author{
Inese Gobina', Una Kojalo', Santa Pildava² \\ 'Riga Stradins University, Latvia \\ ${ }^{2}$ Center of Disease Prevention and Control, Latvia
}

\begin{abstract}
ADRRESS FOR CORRESPONDENCE: Inese Gobin,a, Department of Public Health and Epidemiology, Riga Stradinš University, Riga, Latvia; e-mail: inese.gobina@rsu.Iv
\end{abstract}

Supplementary materials (slides from the conference) are available in Webbappendix at the Journal's website: https://www.termedia.pl/ Journal/Journal_of_Health_Inequalities-100

Although the life expectancy has increased significantly since 2000 in Latvia, it remains one of the lowest in the EU. In 2020, life expectancy at birth was 70.4 years for males and 79.7 for females. The gender gap of almost more than nine years in life expectancy far exceeds the EU average. In 2018, in the EU, males in Latvia had the highest standardised death rate for preventable diseases (548.9 per 100,000), more than three times exceeding this mortality rate for females ( 158.5 per 100,000$)$. This study aimed to explore the gender differences in trends in life expectancy, and leading causes of preventable deaths in the EU were examined in Latvia from 2000 to 2020 for people under 75 years.

The Central Statistical Bureau's official data was used to investigate life expectancy. The national database of Causes of Death from the Centre for Disease Prevention and Control was used to study the all-cause and cause-specific mortality trends from lung cancer, chronic obstructive pulmonary disorders (COPDs), ischaemic heart diseases, cerebrovascular diseases, intentional selfharm, accidental injuries, transport accidents, and alcohol-related disorders and poisonings for people under 75 years old. For the mortality categories analysed, ICD10 codes from the 2018 joint OECD/Eurostat list of preventable and curable causes of death were used.

The trends of life expectancy and preventable mortality rates between males and females were studied with the joinpoint regression analysis. The annual percentage change (APC) and the average annual percentage change (AAPC) in mortality rates with the corresponding 95\% confidence interval (CI) were estimated. The two-sided $p$-value threshold was set at 0.05 .
Between 2000 and 2020, life expectancy increased significantly for males from 64.6 years to 70.4 years $(\mathrm{AAPC}=0.5$; CI 95\%: $0.3-0.6)$ and females from 75.8 to 79.5 years $(\mathrm{APPC}=0.3$; CI 95\%: 0.2-0.3).

From 2000 to 2020 , all-cause mortality declined significantly for both genders (AAPC $=-1.3 ; p<0.05$ ), with the greatest decline occurring from 2006 to 2014. Lung cancer mortality had decreased for males (APPC $=-1.4$; CI 95\%: $-1.8-1.1$ ), whereas increased for females (AAPC $=1.8$; CI 95\%: 1.1-1.6); and the gender mortality rate difference decreased from seven to four times. Mortality rate of COPDs decreased significantly for females (AAPC $=-1.3$; CI 95\%: -2.3 to -0.3 ) but not significantly for males. Premature mortality from ischaemic heart diseases, cerebrovascular diseases, accidental injuries, intentional self-harm, and transport accidents declined significantly for both genders, but the gender disparity in mortality rates remained high, with males having significantly higher rates than females.

Alcohol-related disorders and poisonings significantly increased for males from 2000 to 2012 (APC $=5.1$; CI 95\%: 3.3-7.0), while for females' mortality increased from 2000 to 2013 (APC = 3.7; CI 95\%: 1.9-5.5), but both genders experienced some subsequent decline.

Existing gender differences in life expectancy and preventable mortality rates suggest a continuing lack of tailored public health policies to reduce the gender gap in health status in Latvia.

Key words: gender, life expectancy, preventable mortality.

\section{DISCLOSURE:}

The authors report no conflict of interest. 